Case Report

\title{
Identification of a Novel CSNK2A1-PDGFRB Fusion Gene in a Patient with Myeloid Neoplasm with Eosinophilia
}

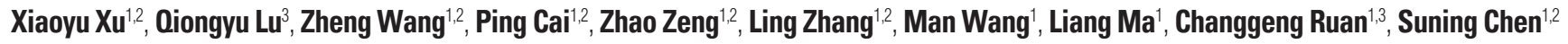 \\ ${ }^{1}$ National Clinical Research Center for Hematologic Diseases, Jiangsu Institute of Hematology, The First Affiliated Hospital of Soochow University, \\ Soochow University, Suzhou, ${ }^{2}$ Institute of Blood and Marrow Transplantation, Collaborative Innovation Center of Hematology, Soochow University, \\ Suzhou, ${ }^{3}$ Cyrus Tang Hematology Center, Soochow University, Suzhou, China
}

Platelet-derived growth factor receptor beta (PDGFRB) rearrangements play an important role in the pathogenesis of eosinophiliaassociated myeloid/lymphoid neoplasms. Up to now, more than 70 PDGFRB fusions have been identified. Here, a novel PDGFRB fusion gene CSNK2A1-PDGFRB has been identified in myeloproliferative neoplasm (MPN) with eosinophilia by RNA-sequencing, which has been verified by reverse transcription polymerase chain reaction and Sanger sequencing. The new PDGFRB fusion partner gene CSNK2A1 encoded one of the two catalytic subunit of casein kinase II (CK2). To our knowledge, this is the first report on the involvement of CSNK2A1 in fusion genes, especially fusion with another kinase PDGFRB in MPN. In addition, the CSNK2A1-PDGFRB fusion retained the entire kinase domain of PDGFRB and response to imatinib at low concentration. The patient with CSNK2A1PDGFRB was sensitive to imatinib treatment and acquired sustained complete remission.

Key words CSNK2A1-PDGFRB, Myeloid neoplasms, RNA-seq, Imatinib

\section{Introduction}

On the base of the 2016 World Health Organization, myeloid/lymphoid neoplasms with eosinophilia are commonly related to rearrangements of PDGFRA, PDGFRB, or FGFR1, or PCM1-JAK2 fusion gene [1]. The PDGFRB gene translocation is one of the most chromosomal aberrant in myeloid neoplasms associated with eosinophil [2], high results in the fusion of the $3^{\prime}$ kinase domain of PDGFRB to a $5^{\prime}$ region of the partner gene. So far more than 70 PDGFRB fusions have been reported, mostly reported in single case. Imatinib mesylate function as a tyrosine kinase inhibitor which can potently inhibit ABL kinase, which is equally against PDGFRB kinase, even at a low concentration [3-5]. Most of the patients with PDGFRB fusions show an outstanding long-term response to imatinib treatment at sub-micromolar concentrations [6].

Casein kinase II (CK2) is ubiquitously expressed, constitutively active serine/threonine protein kinase, which was involved in various cellular processes, including cell growth, survival, apoptosis, and circadian rhythm [7]. CK2 upregulated in a lot of malignancies including hematological cancers [8]. The CK2 tetramer consists of two catalytic CK2 $\alpha$ and $C K 2 \alpha^{\prime}$ subunits, as well as two regulatory CK2 $\beta$ subunits, with the composed patterns of $\alpha 2 \beta 2, \alpha^{\prime} 2 \beta 2$ or $\alpha \alpha^{\prime} \beta 2$. CK2 $\alpha$ was encoded by CSNK $2 A 1$ gene (casein kinase II subunit $\alpha$ ), which

\section{Correspondence: Suning Chen}

Jiangsu Institute of Hematology, The First Affiliated Hospital of Soochow

University, Shizi Street 188, Suzhou 215006, China

Tel/Fax: 86-512-67780441 E-mail: chensuning@sina.com

Received December 2, 2020 Accepted December 23, 2020

Published Online December 24, 2020 was predominantly studied likely because of its ubiquitous nature. But $C K 2 \alpha^{\prime}$ expressed varied, particularly in brain [9]. All domains of CK2 $\alpha$ are highly conserved throughout evolution, but CK2 has low homology with other kinases [10]. In addition, $C K 2 \alpha$ knock out mice are lethal at E11 with multiple embryonic alterations [11]. Therefore, the importance and uniqueness of $C K 2 \alpha$ were highlighted. Here, we reported a new fusion gene involving PDGFRB and CSNK2A1 in a patient with myeloproliferative neoplasm (MPN), who is extremely sensitive to imatinib mesy-late treatment. So far, this is the first report on the involvement of CSNK2A1 in fusion genes in cancers.

\section{Case Report}

A 37-year-old man was admitted to local hospital with weight loss, night sweat, repeating fever for a week in April 2018. The patient's initial laboratory examination showed that leukocyte count was $12.48 \times 10^{9} / \mathrm{L}$ with $37 \%$ eosinophilia in the peripheral blood, hemoglobin concentration was 127 $\mathrm{g} / \mathrm{L}$ and platelet count was $146 \times 10^{9} / \mathrm{L}$. The initial bone marrow (BM) aspirates and biopsy showed hyper leukocytes and significantly increased eosinophils (8\%), and neutrophil alkaline phosphatase score was 18 (Fig. 1A and B). Above all,

Co-correspondence: Changgeng Ruan

Jiangsu Institute of Hematology, The First Affiliated Hospital of Soochow

University, Shizi Street 188, Suzhou 215006, China

Tel/Fax: 86-512-67780441 E-mail: changgengruan@hotmail.com 
A
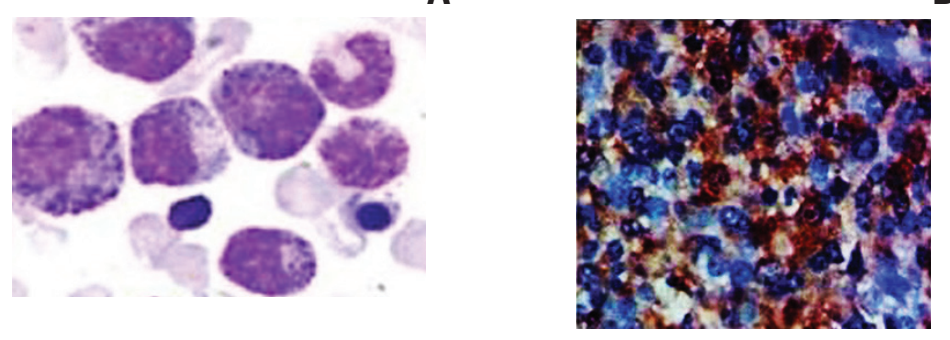

CSNK2A1 (NM 177559.3 exon 4)

PDGFRB (NM 002609.4 exon 12)

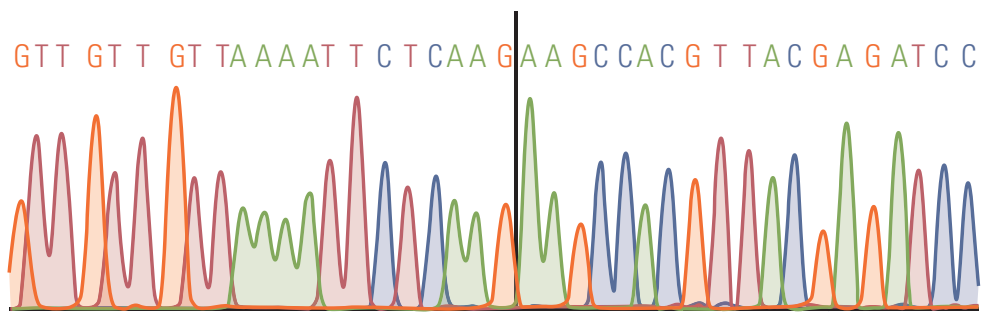

D
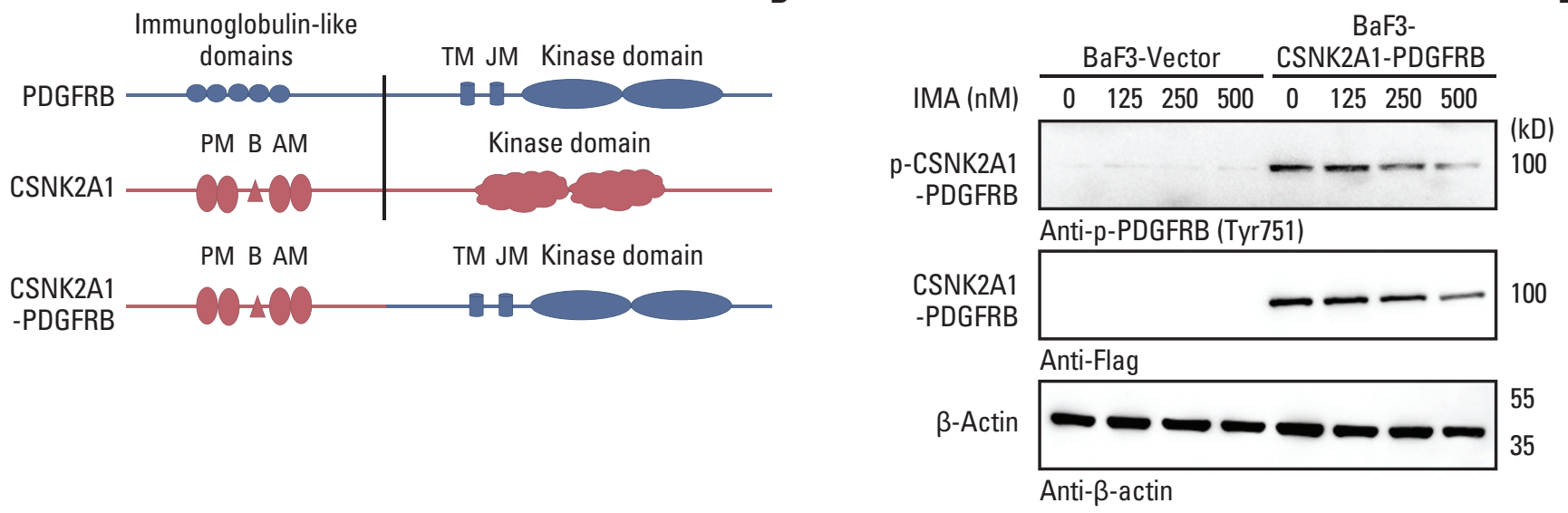

Fig. 1. Identification of novel CSNK2A1-PDGFRB fusions. (A) May-Grünwald-Giemsa staining showing several abnormal eosinophilia in the diagnostic bone marrow aspirate. (B) PDGFRB was stained in bone marrow of patient using immunohistochemistry. (C) Sanger sequencing revealed the fusion between exon 4 of the CSNK2A1 gene (NM_177559.3) and exon 12 of the PDGFRB gene (NM_002609.4). (D) Fusion model of CSNK2A1-PDGFRB are shown. (E) Immunoblot analysis show CSNK2A1-PDGFRB is constitutively activated and is inhibited by imatinib in a concentration-dependent manner. AM, ATP binding domain; B, CK2B subunit binding domain; IMA, imatinib; JM, juxtamembrane domain; PM, polypeptide binding domain; TK, tyrosine kinase domain; TM, transmembrane domain.

these inspections were consistent with a diagnosis of MPN. Then the patient was administrated with $20 \mathrm{mg}$ prednisone per day, but it showed no any effect with white blood cell (WBC) $15.1 \times 10^{9} / \mathrm{L}$, hemoglobin $108 \mathrm{~g} / \mathrm{L}$, platelet $114 \times 10^{9} / \mathrm{L}$, eosinophils $5.13 \times 10^{9} / \mathrm{L}(33.91 \%)$ in peripheral blood.

The suite of fluorescence in situ hybridization (FISH) assay on the BM aspirate was used to detect $B C R-A B L$, PDGFRA, PDGFRB, and FGFR1 rearrangement. MPN FISH assay showed PDGFRB arrangement positive. The karyotype analysis of BM cells showed 46,XY[20].
Patient's RNA was extracted from BM cell by Trizol methods according to the manufacturer's protocol (Invitrogen, Waltham, MA) for RNA-sequencing (RNA-seq) in July 2018. RNA quality and concentration were estimated by Nano Drop ND-2000 (Thermo Fisher Scientific, Waltham, MA). Paired-end reads were generated from the complementary DNA (cDNA) libraries using an Illumina Next Seq 550 instrument (Illumina, San Diego, CA). Then we used starfusion software to analyze the RNA-seq raw data [12] (Supplementary Material). Standard settings were applied for all 
three tools and reads were aligned to the Genome Reference Consortium Human Build 37 (GRCh37). RNA-seq revealed that PDGFRB fused with CSNK2A1 gene. To confirm the fusion, reverse transcription-polymerase chain reaction (RT-PCR) was performed with CSNK2A1-PDGFRB forward primer: 5'-GTGCCAAGCAGGGCCAGAGT-3', reverse primer 5'-AGGGTGCGTCCCAGCACAAG-3'. The reciprocal PDGFRB-CSNK2A1 fusion forward primer: 5'-TCAGAGCTGACACTGGTTCG-3', reverse primer: 5'-GATGTTGGGACCTCCTCTCAA- ${ }^{\prime}$. The PCR products was purified with PCR purification kit (Tiangen, Beijing, China) and sequenced by GENEWIZ Biotechnology Co., Ltd. (Suzhou, Jiangsu, China). The sequence was analyzed using the BLAST program (https://blast.ncbi.nlm.nih.gov/Blast).

Given the existence of PDGFRB fusion, he received imatinib therapy with $200 \mathrm{mg}$ every day orally in September 2018. Two months later, the laboratory examination showed that WBC was $6.14 \times 10^{9} / \mathrm{L}$ with $2.8 \%$ eosinophilia in the peripheral blood (S1 Table). Finally, real-time quantitative reverse transcription PCR was performed to quantify the fusion gene and showed negative in 8 months after imatinib treatment. To date, the patient acquired sustained molecular complete remission for 2 years until the last follow-up.

\section{Discussion}

RNA targeted capture sequencing showed a fusion between CSNK2A1 exon 4 (NM_177559.3) and PDGFRB exon 12 (NM_002609.4), forming a novel fusion gene CSNK2A1PDGFRB. RT-PCR and Sanger sequencing has confirmed CSNK2A1-PDGFRB fusion transcripts (Fig. 1C). The reciprocal fusion transcript PDGFRB-CSNK2A1 was negative by detection of RT-PCR. To our knowledge, this is the first case on CSNK2A1 gene rearrangement in neoplasms, especially fusion with another kinase PDGFRB in hematological cancers. The fusion protein retained the transmembrane domain and the entire kinase domain of PDGFRB (Fig. 1D).

Whole cDNA in CSNK2A1-PDGFRB open reading frame was cloned and transduced to BaF3 cells. As a result, $C S N$ K2A1-PDGFRB fusion protein is constitutively activated (Fig. $1 \mathrm{E}$, panel 5). In addition, incubation of $\mathrm{BaF} 3$ cells transduced with CSNK2A1-PDGFRB with imatinib for 4 hours caused a concentration-dependent decrease of CSNK2A1-PDGFRB protein (Fig. 1E). The result suggested that imatinib might induce the degradation of PDGFRB fusion protein besides inhibition of its activation.

It is well known that dimerization results in activation of PDGFRB and its down signaling play a vital role in mitogenesis, cytoskeletal rearrangements, and chemotaxis [13]. Most partners have coiled-coil domains, which are required for dimerization or oligomerization of $P D G F R B$ fusions. However, there is no coiled-coil motif in $C K 2 \alpha$. In addition, lacking transmembrane domain or disrupting the WW-like domain in juxta membrane region of $P D G F R B$ may also play a role in PDGFRB kinase activation and transformation properties. However, the fusion protein retained the transmembrane domain of PDGFRB. So that, there may be another unknown way to active kinase region of PDGFRB. Notably, CK2 $\alpha$ harbored several regions referred to polypeptide binding domain and $C K 2 \beta$ binding domain, which were retained in the CSNK2A1-PDGFRB fusion, and may be associated with dimerization or oligomerization of the CSNK2A1-PDGFRB fusion. Indeed, the kinase domain of CSNK2A1-PDGFRB was constitutively activated as shown in Fig. $1 \mathrm{E}$ and the patient with CSNK2A1-PDGFRB was sensitive to imatinib treatment.

Totally, we have identified a novel PDGFRB fusion gene with $C K 2 \alpha$ in an MPN by RNA-seq, which was extremely sensitive to imatinib. To our knowledge, it is the first report to find a CK2 $\alpha$ rearrangement in neoplasm, especially fusion with another kinase PDGFRB in MPN.

\section{Electronic Supplementary Material}

Supplementary materials are available at Cancer Research and Treatment website (https: // www.e-crt.org).

\section{Ethical Statement}

The study was approved by the Ethics Committee of the First Affiliated Hospital of Soochow University (No. 221 of 2019 LSP (application)) and was conducted following the Declaration of Helsinki.

\section{Author Contributions}

Conceived and designed the analysis: Chen S, Zeng Z, Ruan C. Collected the data: Xu X.

Contributed data or analysis tools: Xu X, Lu Q, Wang Z, Cai P, Wang M, Ma L.

Performed the analysis: Xu X, Lu Q, Wang Z, Cai P, Zhang L. Wrote the paper: Xu X, Zeng Z.

\section{Conflicts of Interest}

Conflict of interest relevant to this article was not reported.

\section{Acknowledgments}

This study was supported by grant from the National Key R\&D Program of China (2019YFA0111000), the National Natural Science Foundation of China (81700140, 81873449, 81970142, 81900130, 81970136, 82000132), the Natural Science Foundation of the Jiangsu Higher Education Institution of China (18KJA320005), the Natural Science Foundation of Jiangsu Province (BK20190180), China Postdoctoral Science Foundation (2018M632372), the priority academic program development of Jiangsu Higher Education Institution, Translational Research Grant of NCRCH (2020WSB11, 2020WSB13). 


\section{References}

1. Arber DA, Orazi A, Hasserjian R, Thiele J, Borowitz MJ, Le Beau MM, et al. The 2016 revision to the World Health Organization classification of myeloid neoplasms and acute leukemia. Blood. 2016;127:2391-405.

2. Naumann N, Schwaab J, Metzgeroth G, Jawhar M, Haferlach C, Gohring G, et al. Fusion of PDGFRB to MPRIP, CPSF6, and GOLGB1 in three patients with eosinophilia-associated myeloproliferative neoplasms. Genes Chromosomes Cancer. 2015;54:762-70.

3. Apperley JF, Gardembas M, Melo JV, Russell-Jones R, Bain BJ, Baxter EJ, et al. Response to imatinib mesylate in patients with chronic myeloproliferative diseases with rearrangements of the platelet-derived growth factor receptor beta. $\mathrm{N}$ Engl J Med. 2002;347:481-7.

4. Gotlib J. World Health Organization-defined eosinophilic disorders: 2017 update on diagnosis, risk stratification, and management. Am J Hematol. 2017;92:1243-59.

5. Jawhar M, Naumann N, Schwaab J, Baurmann H, Casper J, Dang TA, et al. Imatinib in myeloid/lymphoid neoplasms with eosinophilia and rearrangement of PDGFRB in chronic or blast phase. Ann Hematol. 2017;96:1463-70.

6. Cheah CY, Burbury K, Apperley JF, Huguet F, Pitini V, Gar- dembas $\mathrm{M}$, et al. Patients with myeloid malignancies bearing PDGFRB fusion genes achieve durable long-term remissions with imatinib. Blood. 2014;123:3574-7.

7. Dmytruk KV, Sybirnyi AA. Features and functional characteristics of protein kinase CK2. Ukr Biokhim Zh (1999). 2006;78:27-36.

8. Ahmad KA, Wang G, Slaton J, Unger G, Ahmed K. Targeting CK2 for cancer therapy. Anticancer Drugs. 2005;16:1037-43.

9. Ceglia I, Flajolet M, Rebholz H. Predominance of CK2alpha over CK2alpha' in the mammalian brain. Mol Cell Biochem. 2011;356:169-75.

10. Pinna LA. Casein kinase 2: an 'eminence grise' in cellular regulation? Biochim Biophys Acta. 1990;1054:267-84.

11. Buchou T, Cochet C. Protein kinase CK2: an enzyme that likes to be different. Med Sci (Paris). 2003;19:709-16.

12. Jang YE, Jang I, Kim S, Cho S, Kim D, Kim K, et al. ChimerDB 4.0: an updated and expanded database of fusion genes. Nucleic Acids Res. 2020;48:D817-24.

13. Shang Q, Zhao L, Wang X, Wang M, Sui SF, Mi LZ. Expression and purification of functional PDGF receptor beta. Biochem Biophys Res Commun. 2017;489:353-9. 